\title{
Erratum to: Microsatellite records for volume 7, issue 4
}

\author{
Rose Komugisha Basiita ${ }^{13,14}$. J. Henrich Bruggemann ${ }^{15,16} \cdot$ Nianhui Cai $^{33}$. \\ Conchi Cáliz-Campal $^{8}$ - Chen Chen $^{12} \cdot$ Jiawei Chen ${ }^{19,20} \cdot$ L. Cizek $^{4,5}$. \\ Pedro J. Cordero ${ }^{8}$ - Deborah A. Dawson ${ }^{11}$ - Yandong Ding ${ }^{19,20} \cdot$ L. Drag $^{4,5}$. \\ Anan Duan ${ }^{33} \cdot$ Bruno Fogliani $^{31} \cdot$ Tian-xiang Gao ${ }^{24} \cdot$ Pauline Gélin $^{15,16}$. \\ Martin J. Genner ${ }^{6}$ - Zhi-Min Gu ${ }^{35}$. Mireille M. M. Guillaume ${ }^{15,16,18}$. \\ Jian-Lin Guo $^{35}$ - Chengzhong $\mathrm{He}^{33,34}$ - Peter M. Hollingsworth ${ }^{29}$ - Gavin J. Horsburgh ${ }^{11}$ • \\ Miho Inoue-Murayama ${ }^{25,26} \cdot$ Hideyuki Ito $^{25}$ - Dean Robert Jerry ${ }^{13}$. \\ Yong-Yi Jia ${ }^{35}$ - Wen-Ping Jiang ${ }^{35}$ - Catherine S. Jones ${ }^{10}$ • David Byron Jones ${ }^{13}$. \\ Lingfeng Kong ${ }^{12} \cdot \mathrm{Qi} \mathrm{Li}^{12}$ - Chun-hou $\mathrm{Li}^{23} \cdot \mathrm{Xi}-\mathrm{Lian} \mathrm{Li}^{35} \cdot \mathrm{Jiang} \mathrm{Li}^{37}$.

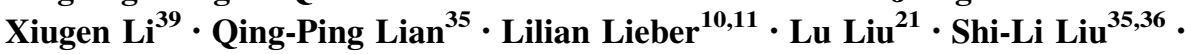 \\ Mengwen $\mathrm{Liu}^{37} \cdot$ Shuping $\mathrm{Luo}^{38} \cdot$ Taku Maeda $^{27} \cdot$ Hélène Magalon ${ }^{15,16}$. \\ Renee M. Martin ${ }^{32}$ - Vincent Mehn ${ }^{15} \cdot$ K. Meyza ${ }^{3}$ Leslie R. Noble ${ }^{10}$. \\ Víctor Noguerales $^{8} \cdot$ Rob Ogden $^{25,28} \cdot$ A. Oleksa $^{3} \cdot$ Manabu Onuma ${ }^{26}$. \\ Joaquín Ortego ${ }^{8,9} \cdot$ Ying Pan $^{1,2} \cdot$ Morgan L. Robinson ${ }^{32}$ - Clément Rougeux ${ }^{15,17}$.

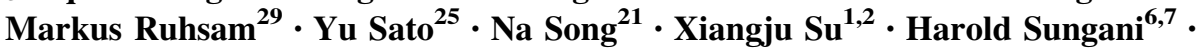

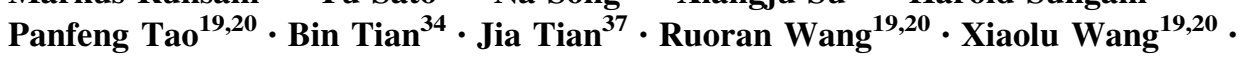 \\ Xiaojun Wang ${ }^{19,20}$ - Dawei Wang ${ }^{33,34}$ - Wade D. Wilson ${ }^{32}$ - Mingcan $\mathrm{Wu}^{1,2}$. \\ Xueping $\mathrm{Wu}^{1,2} \cdot$ Adrien $\mathrm{S}$. Wulff ${ }^{30,31} \cdot$ Yulan $\mathrm{Xu}^{33,34} \cdot$ Yang $\mathrm{Xu} \mathrm{u}^{33,34}$. \\ Takashi Yanagimoto ${ }^{22} \cdot$ Shaowu Yin ${ }^{19,20} \cdot$ Hong Yu ${ }^{12} \cdot$ Bin Zeng ${ }^{37}$. \\ Kyall Richard Zenger ${ }^{13} \cdot$ Guosong Zhang $^{19,20} \cdot$ Jin-Liang Zhao ${ }^{36} \cdot$ Yuna Zhou $^{1,2}$ \\ Published online: 4 February 2016 \\ (C) Springer Science+Business Media Dordrecht 2016
}

\section{Erratum to: Conservation Genet Resour (2015) 7:917-944 DOI 10.1007/s12686-015-0493-8}

In the original publication of the article, the author group was published incorrectly. The correct author group is given in this erratum.

The online version of the original article can be found under doi:10.1007/s12686-015-0493-8.

Rose Komugisha Basiita

Basiita.komugisha@my.jcu.edu.au

1 College of Animal Science and Technology, Guangxi University, 100 Daxue Road, Nanning 530004, Guangxi, China

2 Guangxi Colleges and Universities Key Laboratory of Aquatic Healthy Breeding and Nutrition Regulation, 100 Daxue Road, Nanning 530005, Guangxi, China

3 Institute of Experimental Biology, Kazimierz Wielki University, Chodkiewicza 30, 85-064 Bydgoszcz, Poland
4 Faculty of Sciences, University of South Bohemia, Branisovska 31, 37005 Ceske Budejovice, Czech Republic

5 Institute of Entomology, Biology Centre CAS, v. v. i., Branisovska 31, 37005 Ceske Budejovice, Czech Republic

6 School of Biological Sciences, Life Sciences Building, 24 Tyndall Avenue, Bristol BS8 1TQ, UK

7 Fisheries Research Unit, PO Box 27, Monkey Bay, Malawi 
8 Grupo de Investigación de la Biodiversidad Genética y Cultural, Instituto de Investigación en Recursos Cinegéticos IREC (CSIC, UCLM, JCCM), Ronda de Toledo s/n, 13071 Ciudad Real, Spain

9 Department of Integrative Ecology, Estación Biológica de Doñana, EBD-CSIC, Avda. Américo Vespucio s/n, 41092 Seville, Spain

10 Institute of Biological and Environmental Sciences, School of Biological Sciences, University of Aberdeen, Aberdeen AB24 2TZ, Scotland, UK

11 NERC Biomolecular Analysis Facility, Department of Animal and Plant Sciences, University of Sheffield, Western Bank, Sheffield, South Yorkshire S10 2TN, UK

12 Key Laboratory of Mariculture, Ministry of Education, Ocean University of China, Qingdao 266003, China

13 Centre for Sustainable Tropical Fisheries and Aquaculture, College of Marine and Environmental Sciences, James Cook University, Townsville, QLD 4811, Australia

14 National Agricultural Research Organization, National Fisheries Resources Research Institute, Aquaculture Research and Development Center Kajjansi, P.O. Box 530, Kampala, Uganda
UMR ENTROPIE Université de La Réunion/CNRS/IRD 9220, 15 Avenue René Cassin, CS 92003,

97744 St Denis Cedex 09, La Réunion, France

16

Laboratory of Excellence CORAIL, Perpignan, France

7 Present Address: GIROQ, Département de Biologie, Pavillon Vachon, Université Laval, Sainte-Foy, QC, Canada G1K 7P4

18 Département MPA, UMR BOrEA CNRS-MNHN-UPMCIRD-UCBN-UAG, Museum National d'Histoire Naturelle, 43 rue Cuvier, 75005 Paris, France

19 College of Life Sciences, Key Laboratory of Biodiversity and Biotechnology of Jiangsu Province, Nanjing Normal University, Nanjing 210023, Jiangsu, China

20 Co-Innovation Center for Marine Bio-Industry Technology of Jiangsu Province, Lian Yungang 222005, Jiangsu, China

21 Institute of Evolution \& Marine Biodiversity, Ocean University of China, Qingdao, China

22 National Research Institute of Fisheries Science, Fisheries Research Agency, Yokohama, Japan

23 Key Laboratory for Exploitation \& Utilization of Marine Fisheries Resource in South China Sea, Ministry of Agriculture, South China Sea Fisheries Research Institute, Chinese Academy of Fishery Sciences, Guangzhou, China 
24 Fishery College, Zhejiang Ocean University, Zhoushan, Zhejiang, China

25 Wildlife Research Center of Kyoto University, Kyoto 606-8203, Japan

26 National Institute for Environmental Studies, Tsukuba 305-8506, Japan

27 Research Institute for Environmental Science and Public Health of Iwate Prefecture, Morioka 020-0857, Japan

28 Royal Zoological Society of Scotland, Edinburgh EH12 6TS, UK

29 Royal Botanic Garden Edinburgh, 20A Inverleith Row, Edinburgh EH3 5LR, UK

30 SoREco-NC, 57 Route de l'Anse Vata, 98800 Nouméa, New Caledonia

31 Agronomic Institute of New Caledonia, BP 73, Port Laguerre, Païta 98890, New Caledonia

32 Molecular Ecology Laboratory, Southwestern Native Aquatic Resources and Recovery Center, P.O. Box 219, Dexter, NM 88230, USA

33 Southwest Forestry University, Key Laboratory for Forest Genetic and Tree Improvement \& Propagation in Universities of Yunnan Province, Kunming 650224, Yunnan, China
34 Key Laboratory of Biodiversity Conservation in Southwest China, State Forestry Administration, Southwest Forestry University, Kunming 650204, China

35 Agriculture Ministry Key Laboratory of Healthy Freshwater Aquaculture \& Key Laboratory of Freshwater Aquatic Animal Genetic and Breeding of Zhejiang Province, Zhejiang Institute of Freshwater Fisheries, Huzhou 313001, China

36 Key Laboratory of Freshwater Fishery Germplasm Resources, Shanghai Ocean University, Ministry of Agriculture, Shanghai 201306, China

37 Collage of Forestry and Horticultural, Xinjiang Agricultural University, Urumqi 830052, People's Republic of China

38 Collage of Agronomy, Xinjiang Agricultural University, Urumqi 830052, People's Republic of China

39 Zhengzhou Fruit Research Institute, Chinese Academy of Agricultural Sciences, Zhengzhou 450009, People's Republic of China 